\title{
Finite Volume Element Method for Solving the Elliptic Neumann Boundary Control Problems
}

\author{
Quanxiang Wang \\ College of Science, Nanjing Agricultural University, Nanjing, China \\ Email: 15105195936@163.com
}

How to cite this paper: Wang, Q.X. (2020) Finite Volume Element Method for Solving the Elliptic Neumann Boundary Control Problems. Applied Mathematics, 11, 1243-1252. https://doi.org/10.4236/am.2020.1112085

Received: October 7, 2020

Accepted: December 6, 2020

Published: December 9, 2020

Copyright (c) 2020 by author(s) and Scientific Research Publishing Inc. This work is licensed under the Creative Commons Attribution International License (CC BY 4.0).

http://creativecommons.org/licenses/by/4.0/

\begin{abstract}
Solving optimization problems with partial differential equations constraints is one of the most challenging problems in the context of industrial applications. In this paper, we study the finite volume element method for solving the elliptic Neumann boundary control problems. The variational discretization approach is used to deal with the control. Numerical results demonstrate that the proposed method for control is second-order accuracy in the $L^{2}(\Gamma)$ and $L^{\infty}(\Gamma)$ norm. For state and adjoint state, optimal convergence order in the $L^{2}(\Omega)$ and $H^{1}(\Omega)$ can also be obtained.
\end{abstract}

\section{Keywords}

Finite Volume Element, Neumann Boundary Control, Variational Discretization

\section{Introduction}

In this paper, we study the finite volume element method for solving the Neumann boundary control problems governed by elliptic partial differential equations. The following control problem will be considered

$$
\min J(y(x), u(x))=\frac{1}{2} \int_{\Omega}\left|y(x)-y_{\Omega}(x)\right|^{2} \mathrm{~d} x+\frac{\lambda}{2} \int_{\Gamma}|u(x)|^{2} \mathrm{~d} s,
$$

subject to

$$
\left\{\begin{array}{l}
-\nabla \cdot(A(x) \nabla y(x))+c(x) y(x)=f(x), \text { in } \Omega, \\
(A(x) \nabla y(x)) \cdot \boldsymbol{n}=B u(x), \text { on } \Gamma,
\end{array}\right.
$$

where $\Omega$ is a plane polygonal domain with piecewise smooth boundary $\Gamma$, $\boldsymbol{A}(x)=\left\{a_{i j}(x)\right\}$ is a $2 \times 2$ symmetric and uniformly positive matrix, $c(x)>0$ 
is a sufficient smooth function defined on $\Omega, B$ denotes the linear and continuous control operator which makes $B u(x) \in L^{2}(\Gamma), u(x)$ and $f(x)$ have enough regularity so that this problem has a unique solution.

The control problem (1)-(2) has many applications, see for example [1], for a brief review of applications. Finite element method is an important numerical method for the problems of partial differential equations and is widely used in the numerical solution of distributed optimal control problems [2] [3] [4] [5]. Compared with distributed control problems, the Neumann boundary control problem is more difficult. Error estimates for finite element discretization of Neumann boundary control problems governed by elliptic equations are discussed in some publications. In [6], piecewise constant functions are used to discretize the control and obtain the rates of convergence in $L^{2}(\Gamma)$. The continuous piecewise linear functions are used to discretize the control and the corresponding error estimates are obtained in [7]. The variational discretization of Neumann type elliptic optimal control problems are considered by Hinze [8]. Error estimates for the postprocessing approach applied to the Neumann boundary control problems in polyhedral domains are considered [9] [10].

The finite volume element (FVE) method has been one of the most commonly used numerical methods for solving partial differential equations. The main advantage of the method is that the local physical conservation law can be maintained. So it has been extensively used in computational fluid dynamics. We can refer to [11] for groundwater flow, [12] for weather prediction, [13] for shallow water wave, and to [14] for sedimentation problem. However, there are only a few published results on the finite volume element method for the distributed optimal control problems. In [15] [16], the authors discussed distributed optimal control problems governed by elliptic equations by using the finite volume element methods. The variational discretization approach is used to deal with the control and the error estimates are obtained in some norms. In [17], the authors considered the convergence analysis of discontinuous finite volume methods applied to distributed optimal control problems governed by a class of secondorder elliptic equations.

In this paper, we consider the finite volume element method for solving the elliptic Neumann boundary control problems. Firstly, we introduce the Neumann boundary optimal control problems and their optimal conditions. To solve the optimal control problems, the associated FVE schemes are constructed. The variational discretization approach is used to deal with the control, which can avoid explicit discretization of the control and improve the approximation of the control.

The rest of the paper is organized as follows. In Section 2, we introduce some notations and the associated optimality conditions for the Neumann boundary optimal control problems. Section 3 presents the finite volume element schemes for the Neumann boundary optimal control problems. In Section 4, numerical results are presented to illustrate the effectiveness. Brief conclusions are given in Section 5. 


\section{Some Notations and Optimality Conditions}

In the following, we use the following notations for the inner products and norms on $L^{2}(\Omega)$ and $H^{1}(\Omega)$ :

$$
(v, w)=(v, w)_{L^{2}(\Omega)},\|v\|=\|v\|_{L^{2}(\Omega)},\|v\|_{1}=\|v\|_{H^{1}(\Omega)} .
$$

The corresponding weak formulation for (2) is: Find $y \in H^{1}(\Omega)$ such that

$$
a(y(x), v(x))=(f(x), v(x))+(B u(x), v(x))_{\Gamma}, \forall v(x) \in H^{1}(\Omega),
$$

where

$$
a(y(x), v(x))=\int_{\Omega}\left(\sum_{i, j=1}^{2} a_{i j} \frac{\partial y(x)}{\partial x_{j}} \frac{\partial v(x)}{\partial x_{i}}+c(x) y(x) v(x)\right) \mathrm{d} x,
$$

and

$$
(f(x), v(x))=\int_{\Omega} f(x) v(x) \mathrm{d} x,(B u(x), v(x))_{\Gamma}=\int_{\Gamma} B u(x) v(x) \mathrm{d} s .
$$

Now, we consider the following optimal control problem for the state $y(x)$ and the control $u(x)$ :

$$
\min J(y(x), u(x))=\frac{1}{2} \int_{\Omega}\left|y(x)-y_{\Omega}(x)\right|^{2} \mathrm{~d} x+\frac{\lambda}{2} \int_{\Gamma}|u(x)|^{2} \mathrm{~d} x,
$$

over all $H^{1}(\Omega) \times L^{2}(\Gamma)$ subject to elliptic state problem (3) and the control constraints

$$
u_{a}(x) \leq u(x) \leq u_{b}(x), x \in \Gamma,
$$

where $y_{\Omega}(x) \in L^{2}(\Omega)$ is a given desired state, $\lambda \geq 0$ is a regularization parameter and $u_{a}(x) \leq u_{b}(x)$. We define the set of admissible controls by

$$
U_{a d}=\left\{u(x) \in L^{2}(\Gamma): u_{a}(x) \leq u(x) \leq u_{b}(x)\right\},
$$

where $U_{a d}$ is a nonempty, closed and convex subset of $L^{2}(\Gamma)$.

From standard arguments for elliptic equations, we can obtain the following propositions.

Proposition 1 For fixed control $u(x) \in L^{2}(\Gamma)$, and $f \in L^{2}(\Omega)$, the state Equation (3) admits a unique solution $y(x) \in H^{1}(\Omega)$ and the following a priori estimate holds:

$$
\|y(x)\|_{1} \leq C\left(\|f(x)\|+\|B u(x)\|_{L^{2}(\Gamma)}\right) .
$$

Proposition 2 Let $U_{a d}$ be a nonempty, closed, bounded and convex set, $y_{\Omega}$ in $L^{2}(\Omega)$ and $\lambda>0$, then the optimal control problem (6) admits a unique solution $(\bar{y}(x), \bar{u}(x)) \in H^{1}(\Omega) \times L^{2}(\Gamma)$.

This proof follows standard techniques [18].

The adjoint state equation for $\bar{z} \in H^{1}(\Omega)$ is given by

$$
\left\{\begin{array}{l}
-\nabla \cdot\left(\boldsymbol{A}^{\mathrm{T}}(x) \nabla \bar{z}\right)+c(x) \bar{z}(x)=\bar{y}(x)-y_{\Omega}(x), \text { in } \Omega, \\
\left(\boldsymbol{A}^{\mathrm{T}}(x) \nabla \bar{z}(x)\right) \cdot \boldsymbol{n}=0, \text { on } \Gamma,
\end{array}\right.
$$

Proposition 3 The necessary and sufficient optimality conditions for (6) and 
(7) can be expressed as the variational inequality

$$
\left(\lambda \bar{u}(x)+B^{*} \bar{z}(x), u(x)-\bar{u}(x)\right)_{L^{2}(\Gamma)} \geq 0, \forall u(x) \in U_{a d} .
$$

Further, the variational inequality is equivalent to

$$
\bar{u}(x)=P_{\left[u_{a}(x), u_{b}(x)\right]}\left(-\frac{B^{*} \bar{z}(x)}{\lambda}\right),
$$

where $P_{\left[u_{a}(x), u_{b}(x)\right]}(\cdot)=\min \left\{u_{b}(x), \max \left\{u_{a}(x), \cdot\right\}\right\}$ denotes the orthogonal projection in $L^{2}(\Gamma)$ onto the admissible set of the control, and $B^{*}$ is the adjoint operator of $B$.

\section{FVE Method}

Now we describe the finite volume element discretization of the optimal control problem (6). We consider a quasi-uniform triangulation $T_{h}$. Divide $\bar{\Omega}$ into a sum of finite number of small triangles $K$ such that they have no overlapping internal region and a vertex of any triangle does not belong to a side of any other triangle. At last, we can obtain a triangulation such that $\bar{\Omega}=\bigcup_{K \in T_{h}} K$. The set of all nodes are denoted by

$$
N_{h}=\left\{p \mid p \text { is a node of element } K \in T_{h}\right\} .
$$

In addition, we denote by $\Pi(i)$ the index set of those vertices that, along with $x_{i}$, are in some element of $T_{h}$.

The dual partition $T_{h}^{*}$ corresponding to the primal partition $T_{h}$ is constructed as follows: In each element $K \in T_{h}$ consisting of vertices $x_{i}, x_{j}$ and $x_{k}$, take the barycenter $q$ in $K$ and select the midpoint $x_{i j}$ on each of the three edges $\overline{x_{i} x_{j}}$. Then connect $q$ to the points $x_{i j}$ by straight lines $\gamma_{i j}$. In this way, we obtain the dual element $V_{i}$ whose edges are $\gamma_{i j}$. Figure 1 gives a sketch of a dual element centered at a vertex $x_{i}$.

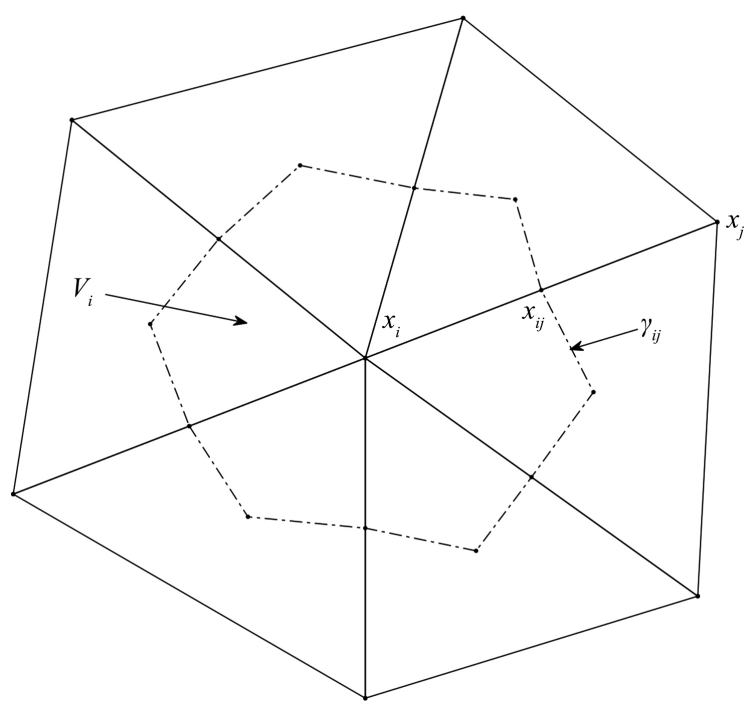

Figure 1. Dual element/control volume with barycenter as internal point. 
We now formulate the discrete form of the optimal control problem. Let $S_{h}$ be the trial function space defined on the triangulation $T_{h}$,

$$
S_{h}=\left\{v \in C(\Omega):\left.v\right|_{K} \text { is linear for all } K \in T_{h}\right\},
$$

and let $Q_{h}$ be the test function space defined on the dual mesh $T_{h}^{*}$,

$$
Q_{h}=\left\{v \in L^{2}(\Omega):\left.v\right|_{V} \text { is constant for all } V \in T_{h}^{*}\right\} .
$$

In this way, we have

$$
S_{h}=\operatorname{span}\left\{\phi_{i}(x) \mid x_{i} \in N_{h}\right\}, Q_{h}=\operatorname{span}\left\{\chi_{i}(x) \mid x_{i} \in N_{h}\right\},
$$

where $\phi_{i}$ are the standard node basis functions with the nodes $x_{i}$, and $\chi_{i}$ are the characteristic functions of the control volume $V_{i}$. Let $I_{h}: C(\Omega) \rightarrow S_{h}$ and $I_{h}^{*}: C(\Omega) \rightarrow Q_{h}$ be the usual interpolation operators, i.e.,

$$
I_{h} y(x)=\sum_{x_{i} \in N_{h}} y\left(x_{i}\right) \phi_{i}(x), I_{h}^{*} y(x)=\sum_{x_{i} \in N_{h}} y\left(x_{i}\right) \chi_{i}(x) .
$$

By the interpolation theorem of Sobolev spaces, we have for $v \in S_{h} \cap H^{2}(\Omega)$ and $w \in S_{h}$

$$
\left|v-I_{h} v\right|_{m} \leq C h^{2-m}|v|_{2}, m=0,1,\left\|w-I_{h}^{*} w\right\| \leq C h|w|_{1} .
$$

Then the finite volume element schemes for (3), (10) and (11) are defined as follows:

$$
\begin{gathered}
a_{h}\left(\bar{y}_{h}, I_{h}^{*} v\right)=\left(f, I_{h}^{*} v\right)+\left(B \bar{u}_{h}, I_{h}^{*} v\right)_{\Gamma}, \forall v \in S_{h}, \\
a_{h}\left(\bar{z}_{h}, I_{h}^{*} w\right)=\left(\bar{y}_{h}-y_{\Omega}, I_{h}^{*} w\right), \quad \forall w \in S_{h}, \\
\left(\lambda \bar{u}_{h}+B^{*} \bar{z}_{h}, u-\bar{u}_{h}\right)_{\Gamma} \geq 0 \text { or } \bar{u}_{h}=P_{\left[u_{a}(x), u_{b}(x)\right]}\left(-\frac{B^{*} \bar{z}_{h}}{\lambda}\right), \forall u \in U_{a d},
\end{gathered}
$$

where

$$
a_{h}\left(\bar{y}_{h}, I_{h}^{*} v\right)=-\sum_{V}\left[I_{h}^{*} v \int_{\partial V_{i}} \boldsymbol{A} \nabla \bar{y}_{h} \cdot \boldsymbol{n} \mathrm{d} s-\int_{V} c_{0} \bar{y}_{h} I_{h}^{*} v \mathrm{~d} x\right] .
$$

We use the following fixed-point iteration algorithm to compute the above discrete optimality problem.

\section{Algorithm 1}

1) Choose $u_{h}^{0} \in U_{a d}$ and set $k=0$;

2) Compute $y_{h}^{k}$ by solving $a_{h}\left(y_{h}^{k}, I_{h}^{*} v\right)=\left(f, I_{h}^{*} v\right)+\left(B u_{h}^{k}, I_{h}^{*} v\right)_{\Gamma}$;

3) Compute $z_{h}^{k}$ by solving $a\left(z_{h}^{k}, I_{h}^{*} w_{h}\right)=\left(y_{h}^{k}-y_{\Omega}, I_{h}^{*} w\right)$;

4) Set $u_{h}^{k+1}=P_{\left[u_{a}(x), u_{b}(x)\right]}\left(-\frac{B^{*} z_{h}^{k}}{\lambda}\right)$;

5) If $\left|u_{h}^{k+1}-u_{h}^{k}\right| \leq 10^{-6}$, then we set $\bar{u}_{h}=u_{h}^{k+1}$, else $k=k+1$, and go back to Step 2 . 


\section{Numerical Examples}

In this section, we report some numerical results of finite volume element schemes for the Neumann boundary optimal control problems. To illustrate the theoretical analysis, the following rate of convergence $r$ is defined,

$$
r=\log _{2}\left(\frac{\left\|u_{2 h}-u\right\|}{\left\|u_{h}-u\right\|}\right),
$$

where $u_{h}$ is the numerical solution with space step size $h$ and $u$ is the analytical solution.

\subsection{Example 1}

To validate the finite volume element schemes for the solution of elliptic problem with the Neumann boundary condition, test example is needed for which the exact solutions are known in advance. We consider the following problem with inhomogeneous Neumann boundary condition,

$$
\left\{\begin{array}{l}
-\Delta y(x)+c(x) y(x)=f(x), \text { in } \Omega, \\
\nabla y(x) \cdot \boldsymbol{n}=g(x), \text { on } \Gamma,
\end{array}\right.
$$

where $\Omega$ denotes unit square $(0,1) \times(0,1), \boldsymbol{n}$ is the outer unit normal vector. We consider the following exact solution to the above boundary value problem

$$
y(x)=x_{1}^{2}+x_{2}^{2}+x_{1} x_{2} .
$$

In this example, we choose $c(x)=1+x_{1}^{2}+x_{2}^{2}$. The source term $f(x)$ and the boundary condition $g(x)$ can be derived from the exact solution.

We compute the $L^{\infty}, L^{2}$ and $H^{1}$ errors for $y(x)$. They are displayed in Table 1. Examination of the table shows that the error measures of the FVE schemes diminish approximately $h^{2}$ for the error in the $L^{2}$ norm and $h$ in the $H^{1}$ norm, which is optimal from the the viewpoint of polynomial degrees. And the error in the $L^{\infty}$ norm is quasi-optimal.

\subsection{Example 2}

This example is taken from [7]. The optimization problem reads

$$
\min J(y, u)=\frac{1}{2} \int_{\Omega}\left(y-y_{\Omega}\right)^{2} \mathrm{~d} x+\frac{\lambda}{2} \int_{\Gamma} u^{2} \mathrm{~d} s+\int_{\Gamma} e_{u}(x) u \mathrm{~d} s+\int_{\Gamma} e_{y} y \mathrm{~d} s,
$$

Table 1. Errors results for Example 1.

\begin{tabular}{ccccccc}
\hline$h$ & $L^{\infty}$ error & $r$ & $L^{2}$ error & $r$ & $H^{1}$ error & $r$ \\
\hline $1 / 8$ & $2.1580 \mathrm{E}-02$ & - & $4.9749 \mathrm{E}-03$ & - & $1.5893 \mathrm{E}-01$ & - \\
$1 / 16$ & $6.4194 \mathrm{E}-03$ & 1.75 & $1.2544 \mathrm{E}-03$ & 1.99 & $8.0310 \mathrm{E}-02$ & 0.98 \\
$1 / 32$ & $1.8578 \mathrm{E}-03$ & 1.79 & $3.1446 \mathrm{E}-04$ & 2.00 & $4.0287 \mathrm{E}-02$ & 1.00 \\
$1 / 64$ & $5.2742 \mathrm{E}-04$ & 1.82 & $7.8683 \mathrm{E}-05$ & 2.00 & $2.0163 \mathrm{E}-02$ & 1.00 \\
$1 / 128$ & $1.4757 \mathrm{E}-04$ & 1.84 & $1.9675 \mathrm{E}-05$ & 2.00 & $1.0084 \mathrm{E}-02$ & 1.00 \\
\hline
\end{tabular}


subject to

$$
\left\{\begin{array}{l}
-\Delta y\left((x)+c(x) y(x)=e_{1}(x), \text { in } \Omega,\right. \\
\nabla y(x) \cdot \boldsymbol{n}=e_{2}(x)+u(x), \text { on } \Gamma,
\end{array}\right.
$$

where $\Omega$ denotes unit square $(0,1) \times(0,1), \lambda=1, c(x)=1+x_{1}^{2}-x_{2}^{2}, \quad e_{y}(x)=1$, $y_{\Omega}=x_{1}^{2}+x_{1} x_{2}, \quad e_{1}(x)=-2+\left(1+x_{1}^{2}-x_{2}^{2}\right)\left(1+2 x_{1}^{2}+x_{1} x_{2}-x_{2}^{2}\right)$,

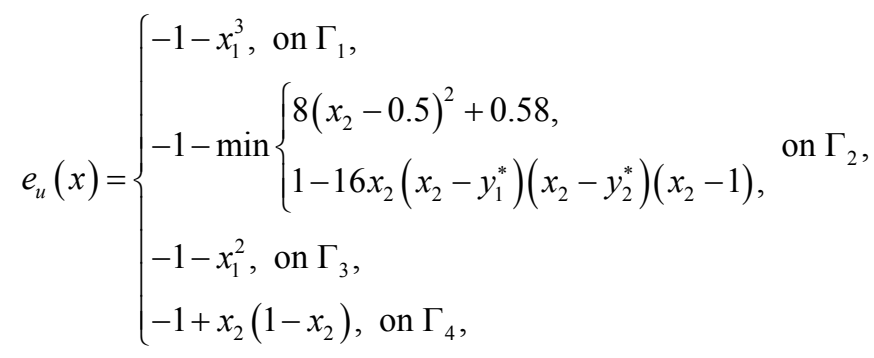

and

$$
e_{2}(x)=\left\{\begin{array}{l}
1-x_{1}+2 x_{1}^{2}-x_{1}^{3}, \text { on } \Gamma_{1}, \\
7+2 x_{2}-x_{2}^{2}-\min \left\{8\left(x_{2}-0.5\right)^{2}+0.58,1\right\}, \text { on } \Gamma_{2}, \\
-2+2 x_{1}+x_{1}^{2}, \text { on } \Gamma_{3}, \\
1-x_{2}-x_{2}^{2}, \text { on } \Gamma_{4},
\end{array}\right.
$$

where $\Gamma_{1}$ to $\Gamma_{4}$ are the four sides of the square, starting at the bottom side and going counterclockwise,

$$
y_{1}^{*}=0.5-\frac{\sqrt{21}}{20} \text { and } y_{2}^{*}=0.5+\frac{\sqrt{21}}{20} .
$$

This problem has the following exact solution: $\bar{y}(x)=1+2 x_{1}^{2}+x_{1} x_{2}-x_{2}^{2}$, $\bar{z}(x)=1$ and

$$
\bar{u}(x)= \begin{cases}x_{1}^{3}, & \text { on } \Gamma_{1}, \\ \min \left\{8\left(x_{2}-0.5\right)^{2}+0.58,1\right\}, & \text { on } \Gamma_{2}, \\ x_{1}^{2}, & \text { on } \Gamma_{3}, \\ 0, & \text { on } \Gamma_{4} .\end{cases}
$$

The convergence results of the control $u(x)$ in Table 2 demonstrate secondorder accuracy in the $L^{2}(\Gamma)$ and $L^{\infty}(\Gamma)$ norm. The $L^{\infty}, L^{2}$ and $H^{1}$ errors for state $y(x)$ and adjoint state $z(x)$ have been computed by the finite volume element schemes. They are displayed in Table 3 and Table 4, respectively. Examination of the tables shows that the error measures of the schemes diminish approximately quadratically for the error in $L^{\infty}$ and $L^{2}$ norm, and linearly for the error in $H^{1}$ norm except the error of adjoint state $z(x)$. This may be due to its better smoothness. Figure 2 shows the computed optimal control with $h=1 / 32$. 
Table 2. Errors of the control for Example 2.

\begin{tabular}{ccccc}
\hline$h$ & $L^{\infty}(\Gamma)$ error & $r$ & $L^{2}(\Gamma)$ error & $r$ \\
\hline $1 / 8$ & $1.4360 \mathrm{E}-03$ & - & $1.4176 \mathrm{E}-03$ & - \\
$1 / 16$ & $3.6011 \mathrm{E}-04$ & 2.00 & $3.5464 \mathrm{E}-04$ & 2.00 \\
$1 / 32$ & $9.0099 \mathrm{E}-05$ & 2.00 & $8.8761 \mathrm{E}-05$ & 2.00 \\
$1 / 64$ & $2.2553 \mathrm{E}-05$ & 2.00 & $2.2030 \mathrm{E}-05$ & 2.01 \\
$1 / 128$ & $5.6702 \mathrm{E}-06$ & 1.99 & $5.5253 \mathrm{E}-06$ & 2.00 \\
\hline
\end{tabular}

Table 3. Errors of the state for Example 2.

\begin{tabular}{ccccccc}
\hline \multicolumn{1}{c}{$h$} & $L^{\infty}$ error & $r$ & $L^{2}$ error & $r$ & $H^{1}$ error & $r$ \\
\hline $1 / 8$ & $1.4335 \mathrm{E}-02$ & - & $4.3446 \mathrm{E}-03$ & - & $1.8989 \mathrm{E}-01$ & - \\
$1 / 16$ & $4.2037 \mathrm{E}-03$ & 1.77 & $1.0918 \mathrm{E}-03$ & 1.99 & $9.5306 \mathrm{E}-02$ & 0.99 \\
$1 / 32$ & $1.2171 \mathrm{E}-03$ & 1.79 & $2.7324 \mathrm{E}-04$ & 2.00 & $4.7710 \mathrm{E}-02$ & 1.00 \\
$1 / 64$ & $3.4726 \mathrm{E}-04$ & 1.81 & $6.8287 \mathrm{E}-05$ & 2.00 & $2.3863 \mathrm{E}-02$ & 1.00 \\
$1 / 128$ & $9.7623 \mathrm{E}-05$ & 1.83 & $1.6953 \mathrm{E}-05$ & 2.01 & $1.1933 \mathrm{E}-02$ & 1.00 \\
\hline
\end{tabular}

Table 4. Errors of the adjoint state for Example 2.

\begin{tabular}{ccccccc}
\hline$h$ & $L^{\infty}$ error & $r$ & $L^{2}$ error & $r$ & $H^{1}$ error & $r$ \\
\hline $1 / 8$ & $1.4360 \mathrm{E}-03$ & - & $5.2125 \mathrm{E}-04$ & - & $2.8099 \mathrm{E}-03$ & - \\
$1 / 16$ & $3.6011 \mathrm{E}-04$ & 2.00 & $1.3011 \mathrm{E}-04$ & 2.00 & $7.0613 \mathrm{E}-04$ & 1.99 \\
$1 / 32$ & $9.0099 \mathrm{E}-05$ & 2.00 & $3.2532 \mathrm{E}-05$ & 2.00 & $1.7677 \mathrm{E}-04$ & 2.00 \\
$1 / 64$ & $2.2553 \mathrm{E}-05$ & 2.00 & $8.1387 \mathrm{E}-06$ & 2.00 & $4.4214 \mathrm{E}-05$ & 2.00 \\
$1 / 128$ & $5.6702 \mathrm{E}-06$ & 1.99 & $2.0439 \mathrm{E}-06$ & 1.99 & $1.1057 \mathrm{E}-05$ & 2.00 \\
\hline
\end{tabular}

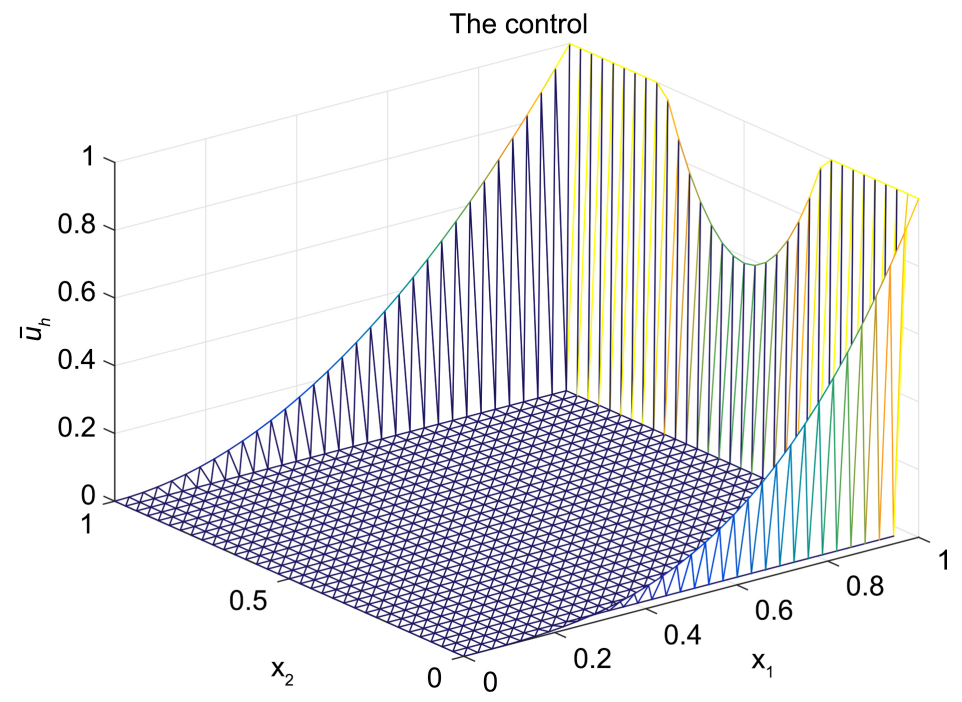

Figure 2. The computed optimal control $\bar{u}_{h}$. 


\section{Conclusion}

In this article, we have considered the finite volume element approximation of the Neumann boundary optimal control problem which is governed by the elliptic partial differential equations. To obtain optimal convergence order, the variational discretization approach is used to deal with the control. Numerical results demonstrate that the proposed FVE method can effectively solve the the Neumann boundary optimal control problem. Second-order accuracy is obtained for the control in the $L^{2}(\Gamma)$ and $L^{\infty}(\Gamma)$ norm. In the future, we will consider the error estimates for the present method.

\section{Acknowledgements}

This project is partially supported by the Fundamental Research Funds for the Central Universities No. KJQN201839.

\section{Conflicts of Interest}

The author declares no conflicts of interest regarding the publication of this paper.

\section{References}

[1] Zhang, Z. (2009) Error Estimates of Finite Volume Element Method for the Pollution in Groundwater Flow. Numerical Methods for Partial Differential Equations, 25, 259-274. https://doi.org/10.1002/num.20340

[2] Arada, N., Casas, E. and Tröltzsch, F. (2002) Error Estimates for the Numerical Approximation of a Semilinear Elliptic Control Problem. Computational Optimization and Applications, 23, 201-229. https://doi.org/10.1023/A:1020576801966

[3] Chen, Y. and Liu, W. (2006) Error Estimates and Superconvergence of Mixed Finite Element for Quadratic Optimal Control. International Journal of Numerical Analysis and Modeling, 3, 311-321.

[4] Casas, E. and Mateos, M. (2008) Error Estimates for the Numerical Approximation of Neumann Control Problems. Computational Optimization and Applications, 39, 265-295. https://doi.org/10.1007/s10589-007-9056-6

[5] Apel, T., Pfefferer, J. and Rösch, A. (2015) Finite Element Error Estimates on the Boundary with Application to Optimal Control. Mathematics of Computation, 84, 33-70. https://doi.org/10.1090/S0025-5718-2014-02862-7

[6] Casas, E., Mateos, M. and Tröltzsch, F. (2005) Error Estimates for the Numerical Approximation of Boundary Semilinear Elliptic Control Problems. Computational Optimization and Applications, 31, 193-219. https://doi.org/10.1007/s10589-005-2180-2

[7] Hou, T. and Li, L. (2016) Error Estimates of Mixed Methods for Optimal Control Problems Governed by General Elliptic Equations. Advances in Applied Mathematics and Mechanics, 8, 1050-10711. https://doi.org/10.4208/aamm.2014.m807

[8] Hinze, M. and Matthes, U. (2009) A Note on Variational Discretization of Elliptic Neumann Boundary Control. Control \& Cybernetics, 38, 577-591.

[9] Pfefferer, J. (2014) Numerical Analysis for Elliptic Neumann Boundary Control Problems on Polygonal Domains. Diss., Univ. der Bundeswehr, Mnchen. 
[10] Becker, R., Kapp, H. and Rannacher, R. (2000) Adaptive Finite Element Methods for Optimal Control of Partial Differential Equations: Basic Concept. SIAM Journal on Control and Optimization, 39, 113-132. https://doi.org/10.1137/S0363012999351097

[11] Tröltzsch, F. (2010) Optimal Control of Partial Differential Equations: Theory, Methods and Applications. American Mathematical Society, Providence.

https://doi.org/10.1090/gsm/112/07

[12] Wang, Q., Zhao, T. and Zhang, Z. (2018) Finite Volume Element Approximation for the Elliptic Equation with Distributed Control. International Journal of Differential Equations, 2018, Article ID: 4753792. https://doi.org/10.1155/2018/4753792

[13] Sandilya, R. and Kumar, S. (2016) Convergence Analysis of Discontinuous Finite Volume Methods for Elliptic Optimal Control Problems. International Journal of Computational Methods, 13, Article ID: 1640012.

https://doi.org/10.1142/S0219876216400120

[14] Wang, Q., Zhang, Z. and Li, Z. (2013) A Fourier Finite Volume Element Method for Solving Two-Dimensional Quasi-Geostrophic Equations on a Sphere. Applied Numerical Mathematics, 71, 1-13. https://doi.org/10.1016/j.apnum.2013.03.007

[15] Apel, T., Winkler, M. and Pfefferer, J. (2018) Error Estimates for the Postprocessing Approach Applied to Neumann Boundary Control Problems in Polyhedral Domains. IMA Journal of Numerical Analysis, 38, 1984-2025.

https://doi.org/10.1093/imanum/drx059

[16] Luo, X., Chen, Y. and Huang, Y. (2013) Some Error Estimates of Finite Volume Element Approximation for Elliptic Optimal Control Problems. International Journal of Numerical Analysis and Modeling, 10, 697-711.

[17] Wang, Q., Zhang, Z., Zhang, X. and Zhu, Q. (2014) Energy-Preserving Finite Volume Element Method for the Improved Boussinesq Equation. Journal of Computational Physics, 270, 58-69. https://doi.org/10.1016/j.jcp.2014.03.053

[18] Ruiz-Baier, R. and Torres, H. (2014) Numerical Solution of a Multidimensional Sedimentation Problem Using Finite Volume-Element Methods. Applied Numerical Mathematics, 95, 280-291. https://doi.org/10.1016/j.apnum.2013.12.006 